\title{
Early warning score immediately prior to discharge predicts good outcome in non-elective medical admissions
}

\author{
Authors: Samuel Thacker, ${ }^{A}$ Tom Bewick ${ }^{B}$ and Joanna Harrison ${ }^{C}$
}

\section{Aims}

To determine whether normal physiological status in a hospitalised adult prior to discharge from an acute NHS trust is correlated with 30-day mortality or 30-day readmission rates.

\section{Methods}

All early warning scores (EWS) from 46,014 consecutive non-elective medical emergency admissions between 1 March 2016 and 1 March 2017 were extracted from electronic records at a large UK teaching hospital, and analysed through Microsoft Excel. Demographic and logistical information including age, Charlson comorbidity score (CCS), length of hospital stay (LOS) and date of death was obtained from the clinical coding department and our Patient Administration System. Episodes were excluded if a) LOS was less than 24 hours or there were fewer than three total measurements of EWS, b) subsequent admissions by the same patient, and c) any episodes where patients died in hospital. A conservative definition for 'stable normal physiology' was created, comprising:

$>$ EWS of zero on at least three consecutive occasions immediately prior to discharge

$>$ no EWS greater than zero for at least 24 hours immediately prior to discharge

$>$ at least one EWS per day over the period during which the maximum EWS remained zero.

The 30-day mortality and 30-day readmission rates were compared between those defined as physiologically normal on discharge and those who were not. The results were stratified by age and CCS groups, and p-values for the chi-squared test were calculated.

Results

After exclusions, 16,381 episodes of care were analysed. Of these patients, 2,519 (15\%) had a physiologically normal status on discharge. There was a statistically significant relationship between stable normal physiology at discharge and a lower 30-day mortality rate $(2.18 \%$ vs $4.40 \%$, unadjusted odds ratio (OR) 0.48 , $95 \%$ confidence interval (CI) $0.37-0.64, \mathrm{p}<0.001)$. This difference was maintained regardless of stratification for age or CCS in those groups where numbers permitted sufficient statistical power (see Table 1). There was a significant association between stable physiological normality and lower 30 -day readmission rate $(11.1 \%$ vs $12.8 \%$, unadjusted OR $0.85,95 \%$ CI $0.75-0.98, p=0.02$ ).

Authors: ${ }^{\mathrm{A}}$ Medicine, ${ }^{\mathrm{B}}$ Respiratory Medicine, ${ }^{\mathrm{C}}$ Information, Derby Teaching Hospitals, Derby, UK
Table 1. 30-day mortality and 30-day readmission rates of patients defined as physiologically normal on discharge and those who were not

\begin{tabular}{|c|c|c|c|c|c|}
\hline $\begin{array}{l}\text { Age } \\
\text { (years) }\end{array}$ & $n$ & $\begin{array}{l}\text { 30-day } \\
\text { mortality } \\
\text { (stable } \\
\text { normal) }\end{array}$ & $\begin{array}{l}\text { 30-day } \\
\text { mortality } \\
\text { (non- } \\
\text { stable } \\
\text { normal) }\end{array}$ & $\begin{array}{l}\text { OR }(95 \% \\
\text { CI) }\end{array}$ & $\begin{array}{l}\text { Chi- } \\
\text { squared } \\
\text { p-value }\end{array}$ \\
\hline$\leq 49$ & 2,993 & $0.24 \%$ & $0.66 \%$ & $\begin{array}{l}0.36 \\
(0.05-2.75)\end{array}$ & 0.31 \\
\hline $50-64$ & 2,909 & $1.07 \%$ & $1.80 \%$ & $\begin{array}{l}0.59 \\
(0.23-1.49)\end{array}$ & 0.26 \\
\hline $65-79$ & 4,808 & $1.80 \%$ & $4.22 \%$ & $\begin{array}{l}0.42 \\
(0.24-0.72)\end{array}$ & 0.001 \\
\hline$\geq 80$ & 5,671 & $4.08 \%$ & $7.87 \%$ & $\begin{array}{l}0.50 \\
(0.35-0.71)\end{array}$ & $<0.001$ \\
\hline \multicolumn{6}{|l|}{ CCS } \\
\hline 0 & 12,706 & $1.75 \%$ & $3.47 \%$ & $\begin{array}{l}0.49 \\
(0.35-0.70)\end{array}$ & $<0.001$ \\
\hline 1 & 2,776 & $2.79 \%$ & $4.63 \%$ & $\begin{array}{l}0.59 \\
(0.31-1.14)\end{array}$ & 0.11 \\
\hline 2 & 615 & $5.19 \%$ & $9.48 \%$ & $\begin{array}{l}0.52 \\
(0.18-1.49)\end{array}$ & 0.22 \\
\hline$\geq 3$ & 284 & $19.23 \%$ & $28.87 \%$ & $\begin{array}{l}0.56 \\
(0.20-1.54)\end{array}$ & 0.26 \\
\hline $\begin{array}{l}\text { Total/ } \\
\text { mean }\end{array}$ & 16,381 & $2.18 \%$ & $4.40 \%$ & $\begin{array}{l}0.48 \\
(0.47-0.64)\end{array}$ & $<0.001$ \\
\hline
\end{tabular}

CCS = Charlson comorbidity score; $\mathrm{CI}=$ confidence interval; $\mathrm{OR}=$ odds ratio

\section{Conclusions}

These results suggest that patients who undergo a sustained period of physiological normality immediately prior to discharge are at lower risk of death or readmission to hospital within 30 days than those who do not. These findings may help reassure clinicians making decisions concerning discharge, and may help determine which patients require earlier follow up or further support.

\section{Conflict of interest statement}

None declared. 